\title{
Annual risk of tuberculosis infection in rural China: a population-based prospective study
}

\author{
Lei Gao ${ }^{1,15,16}$, Liqiong Bai ${ }^{2,15}$, Jianmin Liü3,15, Wei Lư ${ }^{4,15}$, Xinhua Wang ${ }^{5,15}$, \\ Xiangwei $\mathrm{Li}^{1,{ }^{15}}$, Jiang $\mathrm{Du}{ }^{1,15}$, 'Xinchun Chen ${ }^{6,15}$, Haoran Zhang ${ }^{1}$, Henan Xin ${ }^{1}$, \\ Hongtao Sui ${ }^{1}$, Hengjing $\mathrm{Li}^{1}$, Haoxiang $\mathrm{Su}^{1}$, Jian $\mathrm{He}^{5}$, Shouguo Pan ${ }^{7}$, Hong Peng ${ }^{4}$, \\ Zuhui $\mathrm{Xu}^{2}$, Antonino Catanzaro ${ }^{8}$, Thomas G. Evans ${ }^{9}$, Zongde Zhang ${ }^{10}$, Yu Ma ${ }^{10}$, \\ Mufei $\mathrm{Li}^{1}$, Boxuan Feng ${ }^{1}$, Zhen $\mathrm{Li}^{1}$, Ling Guan ${ }^{4}$, Fei Shen ${ }^{4}$, Zhijian Wang ${ }^{11}$, \\ Tao Zhu ${ }^{11}$, Shumin Yang ${ }^{5}$, Hongyan $\mathrm{Si}^{5}$, Yi Wang ${ }^{12}$, Yunhong Tan ${ }^{2}$, \\ Tianzhu Chen ${ }^{13}$, Chen Chen ${ }^{9}$, Yinyin Xia ${ }^{14}$, Shiming Cheng ${ }^{14}$, Weiguo $\mathrm{Xu}^{4,16}$ and \\ Qi Jin ${ }^{1,16}$ for the LATENTTB-NSTM study team
}

Affiliations: ${ }^{1} \mathrm{MOH}$ Key Laboratory of Systems Biology of Pathogens, Institute of Pathogen Biology, and Centre for Tuberculosis, Chinese Academy of Medical Sciences and Peking Union Medical College, Beijing, China. ${ }^{2}$ Hunan Provincial Institute of Tuberculosis Prevention and Control, Changsha, China. ${ }^{3}$ The Sixth People's Hospital of Zhengzhou, Zhengzhou, China. ${ }^{4}$ Jiangsu Provincial Center for Diseases Control and Prevention, Nanjing, China. ${ }^{5}$ Gansu Provincial Center for Diseases Control and Prevention, Lanzhou, China. ${ }^{6}$ Guangdong Key Laboratory for Emerging Infectious Diseases, Shenzhen Third People's Hospital, Shenzhen, China. ${ }^{7}$ Zhongmu County Center for Diseases Control and Prevention, Zhongmu, China. ${ }^{8}$ Dept of Medicine, University of California, San Diego, CA, USA. ${ }^{9}$ Aeras, Rockville, MD, USA. ${ }^{10}$ Beijing Chest Hospital, Capital Medical University, Beijing Key Laboratory for Drug Resistant Tuberculosis Research, Beijing Tuberculosis and Thoracic Tumor Research Institute, Beijing. China. ${ }^{11}$ Danyang City Center for Diseases Control and Prevention, Danyang, China. ${ }^{12}$ Longxi County Center for Diseases Control and Prevention, Longxi, China. ${ }^{13}$ Xiangtan County Center for Diseases Control and Prevention, Xiangtan, China. ${ }^{14}$ Chinese Center for Disease Prevention and Control, Beijing, China. ${ }^{15}$ These authors contributed equally. ${ }^{16}$ These authors contributed equally.

Correspondence: Qi Jin, MOH Key Laboratory of Systems Biology of Pathogens, Institute of Pathogen Biology, and Centre for Tuberculosis, Chinese Academy of Medical Sciences and Peking Union Medical College, No 9 Dong Dan San Tiao, Beijing 100730, China. E-mail: zdsysQvip.sina.com

ABSTRACT Prospective population data on the incidence of tuberculosis (TB) infection has been sparsely reported in the global literature.

A population-based prospective study was conducted in rural China to investigate the annual risk of TB infection, and its persistence using serial tuberculin skin tests (TSTs) and an interferon- $\gamma$ release assay. In total, 13580 eligible participants from four rural sites, identified as TST negative $(<10 \mathrm{~mm})$ or QuantiFERON-TB Gold In-Tube (QFT) (an interferon- $\gamma$ release assay) negative from a baseline survey, were included in the first year's follow-up examination.

The annual conversion rate of QFT among the study sites ranged between $2.1 \%$ and $4.9 \%$ (average $3.1 \%$ ), and the incidence of TST conversion ranged between $6.0 \%$ and $31.1 \%$ (average 14.5\%). During the second year's follow-up, infection persistence was investigated using 390 subjects with QFT conversions. Among them, 49.7\% (164 out of 330) were found to be consistently QFT positive. Both the conversion and the persistence of QFT positivity were found to be significantly increased with increasing age.

In conclusion, the annual $\mathrm{TB}$ infection rate was suggested to be $\sim 1.5 \%$ based on persistent positive results after QFT conversion in rural China. Therefore, infection control among those high-risk populations, including the elderly, should be prioritised for TB control in China.

$@$ ERSpublications

Annual TB infection rate $\sim 1.5 \%$ in rural China, based on persistent positive results after IGRA conversion http://ow.ly/4n95Cw

This article has supplementary material available from erj.ersjournals.com

Received: Jan 312016 | Accepted after revision: April 132016 | First published online: May 262016

Copyright OERS 2016 


\section{Introduction}

Active pulmonary tuberculosis (TB) is caused by infection with Mycobacterium tuberculosis but in $>90 \%$ of infections, the pathogen does not cause disease and is assumed to be in a quiescent state. The risk of active disease is highest in the first 2 years, estimated to be $\sim 5 \%$, followed by an additional $5 \%$ for the remaining lifetime $[1,2]$. Hence, new infections pose the highest risk of disease development and add to the reservoir of chronic latent tuberculosis infection (LTBI). Based on this, preventing transmission of M. tuberculosis should be the first defensive line for TB elimination, which can be augmented through early case finding and prioritisation of prevention among those newly infected [3]. However, given the difference in disease risk of those recently versus chronically infected, different strategies should be applied. As such, the World Health Organization and many developed countries have guidance for close contacts of patients with active TB that prescribe screening for infection status and universal preventive treatment for those with recent infections [4-6].

In 2014, China had the third largest number of incident TB cases (0.9-1.0 million) worldwide [7]. Successful management of TB and addressing LTBI in China are critical for achieving the ambitious targets of the post-2015 Global TB Strategy [8]. However, as suggested by D'Ambrosio et al. [9], to reach TB elimination, sound and bold policies need to be implemented at the national level, and the whole package of necessary core interventions must be duly put into practice. Similarly, good preparedness and proper planning are critical for China to develop feasible and effective strategies. Obtaining a robust estimate of the annual TB infection incidence will enable researchers and public health programmes to evaluate the current status of transmission in a population for the development of tailored risk-based interventions. Therefore, we designed a follow-up survey to address the annual risk of TB infection in rural populations in China.

\section{Methods}

\section{Study design}

This was a multicentre prospective cohort study conducted at four study sites in rural China, organised by the Institute of Pathogen Biology of the Chinese Academy of Medical Sciences and the Chinese Center for Disease Control and Prevention. The study protocol was approved by the ethics committees of the Institute of Pathogen Biology and the Chinese Academy of Medical Sciences.

Between July 1 and September 30, 2013, we conducted a baseline survey, in which participants were screened for LTBI using both tuberculin skin test (TST) and interferon- $\gamma$ release assay (IGRA) [10]. 1 year later, between July 1 and September 30, 2014, the yearly follow-up survey was undertaken with the aim of estimating the incidence of TB infection. We retested the infection status of individuals with negative baseline IGRA and/or TST results ( $<10 \mathrm{~mm}$ as cut-off). A second yearly follow-up survey was undertaken in July, 2015, to estimate the persistence of TB infection among those with QuantiFERON-TB Gold In-Tube (QFT) (Qiagen, Hilden, Germany) conversions. Westat (Rockville, MD, USA) provided independent site monitoring (external quality assessment) according to the procedures and requirements stated in the study protocol.

\section{Study participants}

Registered rural residents at the four study sites (sites A-D) were the target populations of the present and previous study. The study sites were selected based on a wide range of local TB epidemiology, economic conditions and geographic diversity (supplementary table S1) [10]. The inclusion criteria were: birth prior to June 1, 2008 ( $\geqslant 5$ years old); household registration or residence permit for that village; continuous residence at the study site for $\geqslant 6$ months over the past year; ability to complete the investigations and tests during the study duration; and provision of voluntary written informed consent. The exclusion criteria were: current active TB; self-reported history of TB; and pregnancy.

For the first year's follow-up survey, the inclusion criteria were based on the completed study documents from baseline screening (questionnaire and test results including IGRA and TST): not diagnosed with active TB at baseline screening; IGRA negative or TST $<10 \mathrm{~mm}$ at baseline; continuous residence at the study site for $\geqslant 6$ months over the past year; and provision of voluntary written informed consent for the follow-up investigation. The exclusion criteria were outmigration or death during the past year and pregnancy.

For the second year's follow-up survey, the inclusion criteria were IGRA negative for baseline and positive for the first yearly follow-up survey; continuous residence at the study site for $\geqslant 6$ months over the past year. The exclusion criteria were outmigration or death during the past year and pregnancy.

Support statement: This study was supported by the National Science and Technology Major Project of China (grants 2013ZX10003004-002 and 2014ZX10003001-001) and the Program for Changjiang Scholars and Innovative Research Team at the University of China (IRT13007). Funding information for this article has been deposited with FundRef.

Conflict of interest: None declared 


\section{Follow-up procedures}

Details of the baseline survey have been published previously [9]. Based on the baseline result of IGRA and TST, a cohort of infection negatives was defined as: without active TB disease based on absence of TB symptoms and IGRA negative or TST $<10 \mathrm{~mm}$. The first yearly follow-up survey was conducted in parallel at the four study sites among infection negatives during July to September 2014. A standardised questionnaire administered by trained interviewers was completed for each study participant.

Venous blood was collected for all members of the infection negative cohort for QFT testing. QFT was performed as recommended by the manufacturer using a cut-off value of $\geqslant 0.35 \mathrm{IU} \cdot \mathrm{mL}^{-1}$. QFT conversion was defined as a change from negative to positive within 2 years as per 2010 US Centers for Disease Control and Prevention updated guidance on IGRAs [11]. TST placement was done immediately after QFT using the Mantoux method by injecting $0.1 \mathrm{~mL}$ of 5 tuberculin units purified protein derivative (Xiangrui, Beijing, China) intradermally, into the left forearm by preference [12]. Tuberculin reaction size (induration) was measured in millimetres after $48-72 \mathrm{~h}$ of placement by trained study personnel. TST conversion was defined as a change from negative to positive with an increase of $\geqslant 10 \mathrm{~mm}$ in induration within 2 years [5]. Those with evidence of skin disease on their forearms did not undergo TST.

The second yearly follow-up survey was conducted during July 2015 among those with QFT conversion. The QFT was repeated and QFT-conversion persistence was defined as a consistent positive result for both the first and the second yearly follow-up tests.

\section{Data management and statistical analysis}

Questionnaire data and tests results were doubly entered into a spreadsheet and checked by web-based project-specific data collection and management software. After thorough data review, the data were then converted and analysed using Statistical Analysis System 9.2 (SAS Institute Inc., Cary, NC, USA).

Age, sex, occupation and educational level were considered as demographic factors in the analyses. Based on previous reports, history of contact with TB patients, history of immune system disorders, smoking and alcohol drinking status, household per capita income, body mass index (calculated) and number of bacille Calmette-Guérin (BCG) scars were classified as potential factors associated with infection risks. Household per capita income was classified with respect to the national mean level in 2010 (RMB 6000).

Pearson's Chi-squared test and Student's t-test were used to compare the categorical and continuous variables in the study participants, respectively. To identify potential variables related to test conversion and persistence, all variables showing significant relations in univariate analysis were entered into unconditional multiple logistic regression analyses, and the associations were then assessed by means of odds ratios and $95 \%$ confidence intervals. Cohen's $\kappa$ coefficient was calculated to evaluate the agreement between QFT and TST conversions.

As a sensitivity analysis, an alternative definition of QFT conversion (using a cut-off value of $\geqslant 0.70 \mathrm{IU} \cdot \mathrm{mL}^{-1}$ ) was used for the association analysis [13].

\section{Results}

Study participants included in the infection-negative cohort

As shown in table S2, a total of 15907 eligible participants "without active TB and with baseline QFT negative and/or TST $<10 \mathrm{~mm}$ results" were included in the follow-up surveys. Among them, 13580 participated in the first yearly follow-up activities with a response rate of 85.4\% (13580 out of 15907). Table 1 describes the characteristics of the study participants examined in the first yearly follow-up by study site. Overall, $54.6 \%$ (7414 out of 13580 ) of the participants were female, $19.0 \%$ (2573 out of 13580) were younger than 20 years and $23.7 \%$ (3220 out of 13580) were 60 years or older.

\section{TST and QFT conversion}

The 11422 TST-negative $(<10 \mathrm{~mm})$ subjects at baseline were included in the first yearly follow-up investigation and 1654 (14.5\%) of them met the definition of conversion after 1 year (table 1 and figure 1). The TST conversion rate of site A was the highest (31.1\%) and that of site B was the lowest (6.0\%). The distribution of annual TST conversion rates by age, sex and study site is shown in figure 2. Association analyses (table 2) showed TST conversion increased with age ( $\mathrm{p}<0.001$ for trend). History of close contact with pulmonary TB (PTB) patients was identified to be related with an increased risk of TST conversion, with an adjusted odds ratio of 1.27 (95\% CI 1.00-1.61).

As shown in table 1 and figure 1, 12749 QFT-negative subjects at baseline were investigated at the follow-up survey; 390 (3.1\%) of them were found to be positive and $33(0.3 \%)$ had indeterminate results. The QFT conversion rate at 1 year ranged from $2.1 \%$ (site B) to $4.9 \%$ (site C). Individuals with indeterminate results were not included in the association analyses, as shown in table 3. A significantly 
TABLE 1 Characteristics of the study participants and the conversion of the tuberculin skin test (TST) and QuantiFERON-TB Gold In-Tube (QFT)

\begin{tabular}{|c|c|c|c|c|c|c|}
\hline & Total & Site A & Site B & Site C & Site D & Chi-squared $p$-value \\
\hline \multicolumn{7}{|l|}{ Sex } \\
\hline Female & 7414 (54.59\%) & 2072 (55.37\%) & $2002(49.71 \%)$ & $1627(56.05 \%)$ & 1713 (58.91\%) & $<0.001$ \\
\hline Male & $6166(45.41 \%)$ & $1670(44.63 \%)$ & $2025(50.29 \%)$ & $1276(43.95 \%)$ & $1195(41.09 \%)$ & \\
\hline \multicolumn{7}{|l|}{ Age } \\
\hline $5-9$ years & $1207(8.89 \%)$ & $56(1.50 \%)$ & $547(13.58 \%)$ & $156(5.37 \%)$ & 448 (15.41\%) & $<0.001$ \\
\hline $10-19$ years & $1366(10.06 \%)$ & $180(4.81 \%)$ & $429(10.65 \%)$ & $262(9.03 \%)$ & $495(17.02 \%)$ & \\
\hline $20-29$ years & $1052(7.75 \%)$ & $224(5.99 \%)$ & $459(11.40 \%)$ & $161(5.55 \%)$ & $208(7.15 \%)$ & \\
\hline $30-39$ years & $1292(9.51 \%)$ & $306(8.18 \%)$ & $420(10.43 \%)$ & 249 (8.58\%) & $317(10.90 \%)$ & \\
\hline $40-49$ years & $3109(22.89 \%)$ & $897(23.97 \%)$ & $949(23.57 \%)$ & $662(22.80 \%)$ & $601(20.67 \%)$ & \\
\hline $50-59$ years & $2334(17.19 \%)$ & $810(21.65 \%)$ & $576(14.30 \%)$ & $571(19.67 \%)$ & $377(12.96 \%)$ & \\
\hline $60-69$ years & $2081(15.32 \%)$ & 808 (21.59\%) & $403(10.01 \%)$ & 550 (18.95\%) & $320(11.00 \%)$ & \\
\hline$\geqslant 70$ years & 1139 (8.39\%) & $461(12.32 \%)$ & $244(6.06 \%)$ & $292(10.06 \%)$ & $142(4.88 \%)$ & \\
\hline \multicolumn{7}{|l|}{ Highest education level } \\
\hline Primary school or lower & $7580(55.82 \%)$ & $1727(46.15 \%)$ & 2349 (58.33\%) & $1624(55.94 \%)$ & $1880(64.65 \%)$ & $<0.001$ \\
\hline Middle school & $4383(32.28 \%)$ & 1491 (39.85\%) & $123(30.67 \%)$ & $986(33.96 \%)$ & $671(23.07 \%)$ & \\
\hline High school & $1281(9.43 \%)$ & $410(10.96 \%)$ & $5347(8.62 \%)$ & $250(8.61 \%)$ & $274(9.42 \%)$ & \\
\hline College or higher & $336(2.47 \%)$ & $114(3.05 \%)$ & $96(2.38 \%)$ & $43(1.48 \%)$ & $83(2.85 \%)$ & \\
\hline \multicolumn{7}{|l|}{ Household per capita income } \\
\hline Less than RMB 6000 & $9099(67.00 \%)$ & $1583(42.30 \%)$ & $3232(80.26 \%)$ & $1939(66.79 \%)$ & $2345(80.64 \%)$ & $<0.001$ \\
\hline RMB 6000 or more & 4481 (33.00\%) & 2159 (57.70\%) & 795 (19.74\%) & 964 (33.21\%) & $563(19.36 \%)$ & \\
\hline \multicolumn{7}{|l|}{ Smoking status } \\
\hline Never smoked & $10504(77.37 \%)$ & $2891(77.26 \%)$ & $3056(75.94 \%)$ & 2099 (72.30\%) & $2458(84.53 \%)$ & $<0.001$ \\
\hline Ever smoked & $3073(22.63 \%)$ & $851(22.74 \%)$ & $968(24.06 \%)$ & $804(27.70 \%)$ & 450 (15.47\%) & \\
\hline \multicolumn{7}{|l|}{ Alcohol drinking } \\
\hline No & $11002(81.02 \%)$ & 2459 (84.71\%) & 2638 (90.72\%) & $2911(77.79 \%)$ & 2994 (74.37\%) & $<0.001$ \\
\hline Yes & $2577(18.98 \%)$ & 444 (15.29\%) & $270(9.28 \%)$ & $831(22.21 \%)$ & $1032(25.63 \%)$ & \\
\hline \multicolumn{7}{|l|}{ BMI } \\
\hline$<18.5 \mathrm{~kg} \cdot \mathrm{m}^{-2}$ & $6244(45.98 \%)$ & $2028(54.20 \%)$ & 1483 (36.83\%) & $1460(50.31 \%)$ & 1273 (43.78\%) & $<0.001$ \\
\hline$\geqslant 18.5-<24 \mathrm{~kg} \cdot \mathrm{m}^{-2}$ & $2366(17.42 \%)$ & $236(6.31 \%)$ & 775 (19.25\%) & $477(16.44 \%)$ & 878 (30.19\%) & \\
\hline$\geqslant 24-<28 \mathrm{~kg} \cdot \mathrm{m}^{-2}$ & $3587(26.42 \%)$ & $1155(30.87 \%)$ & $1104(27.41 \%)$ & $730(25.16 \%)$ & $598(20.56 \%)$ & \\
\hline$\geqslant 28 \mathrm{~kg} \cdot \mathrm{m}^{-2}$ & $1382(10.18 \%)$ & $323(8.63 \%)$ & $665(16.51 \%)$ & $235(8.10 \%)$ & $159(5.47 \%)$ & \\
\hline \multicolumn{7}{|l|}{ Number of BCG scars } \\
\hline 0 & $6423(47.30 \%)$ & $2523(67.42 \%)$ & $747(18.55 \%)$ & $650(22.40 \%)$ & $2503(86.07 \%)$ & $<0.001$ \\
\hline 1 & $4681(34.47 \%)$ & $937(25.04 \%)$ & 1773 (44.03\%) & $1568(54.03 \%)$ & $403(13.86 \%)$ & \\
\hline$\geqslant 2$ & 2475 (18.23\%) & $282(7.54 \%)$ & 1507 (37.42\%) & $684(23.57 \%)$ & $2(0.07 \%)$ & \\
\hline \multicolumn{7}{|c|}{ History of close contact with TB patient } \\
\hline No & 12903 (95.17\%) & 3685 (98.48\%) & 3864 (95.95\%) & 2491 (85.81\%) & 2863 (99.20\%) & $<0.001$ \\
\hline Yes & $655(4.83 \%)$ & 57 (1.52\%) & $163(4.05 \%)$ & $412(14.19 \%)$ & $23(0.80 \%)$ & \\
\hline \multicolumn{7}{|l|}{ TST } \\
\hline Negative & $9768(85.52 \%)$ & $1931(68.92 \%)$ & 3528 (93.38\%) & 1740 (82.50\%) & $2569(94.00 \%)$ & $<0.001$ \\
\hline Positive & 1654 (14.48\%) & 871 (31.08\%) & $250(6.62 \%)$ & $369(17.50 \%)$ & $164(6.00 \%)$ & \\
\hline \multicolumn{7}{|l|}{ QFT } \\
\hline Negative & $12326(96.68 \%)$ & 3394 (97.08\%) & $3629(97.84 \%)$ & 2652 (94.44\%) & $2651(96.89 \%)$ & $<0.001$ \\
\hline Positive & $390(3.06 \%)$ & $95(2.72 \%)$ & $78(2.10 \%)$ & 138 (4.91\%) & 79 (2.89\%) & \\
\hline Indeterminate & $33(0.26 \%)$ & $7(0.20 \%)$ & $2(0.05 \%)$ & $18(0.64 \%)$ & $6(0.22 \%)$ & \\
\hline
\end{tabular}

Data may not sum to total due to missing data. No significant difference on frequency of missing data was observed between sites. BMI: body mass index; BCG: bacille Calmette-Guérin;

TB: tuberculosis. 


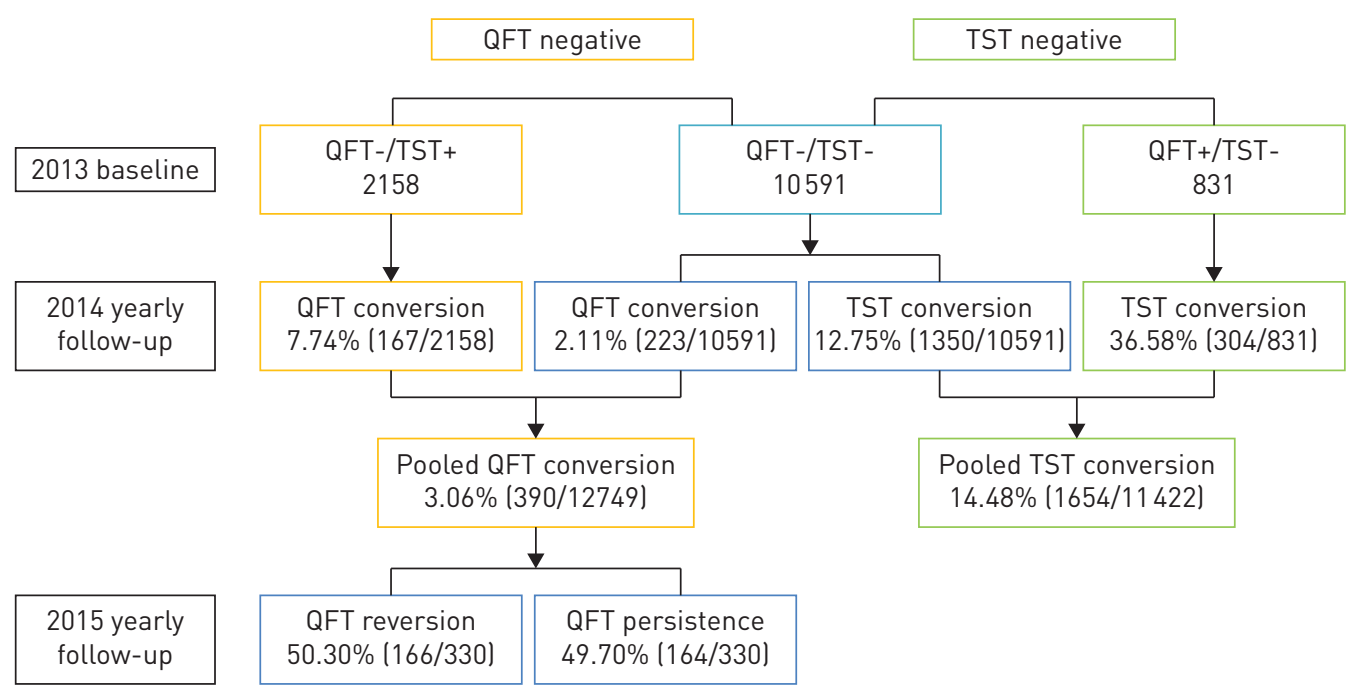

FIGURE 1 The acquisition and persistence of tuberculosis infection among study participants. The conversion and its persistence of QuantiFERON-TB Gold In-Tube (QFT) and tuberculin skin test (TST) are shown by these two tests' results at baseline.

higher incidence of QFT conversion was found for males than females. An increased trend of QFT conversions was observed with increasing age, with the highest incidence shown among participants older than 69 years (4.5\%). The distribution of annual QFT conversion rate by age, sex and study site was shown in figure 2. A history of close contact with PTB patients was identified to be associated with an increased risk of QFT conversion with an adjusted odds ratio of 1.53 (95\% CI 1.03-3.25).

Furthermore, the conversion analysis was performed using participants having negative results for both QFT and TST at baseline. The QFT conversion rate at 1 year ranged from $1.7 \%$ (59 out of 3460, site B) to $2.7 \%$ (54 out of 2014, site C) and TST conversion rates ranged from $4.3 \%$ (111 out of 2561 , site D) to $28.3 \%$ (724 out of 2556, site A). The agreement between QFT and TST conversions were assessed in this population as well, a total of 10563 participants were considered in the analyses after excluding "indeterminate" results
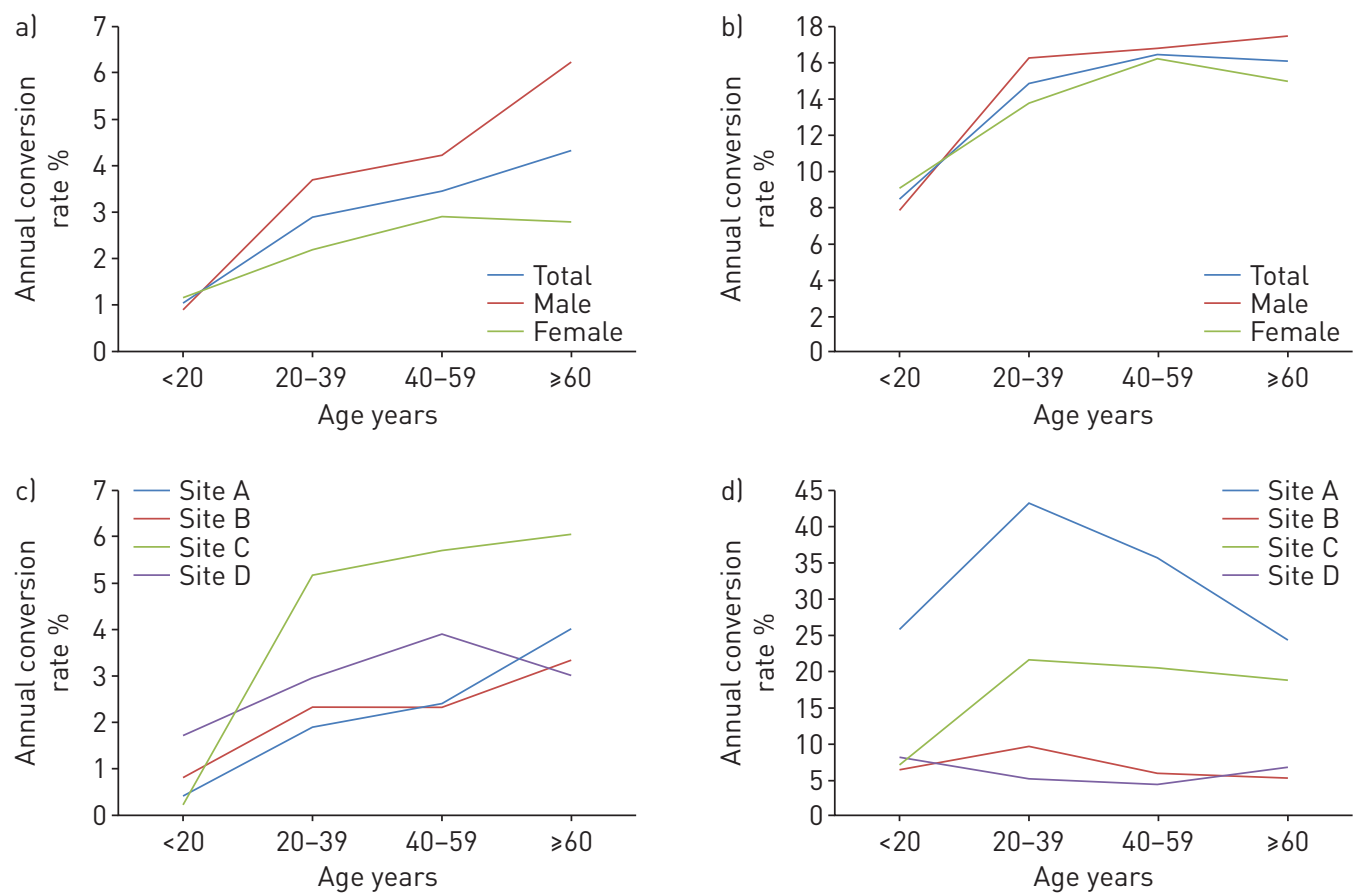

FIGURE 2 Annual QuantiFERON-TB Gold In-Tube (QFT) and tuberculin skin test (TST) conversion rates by age, sex and study site. Due to limited the number of conversions, age group is shown in 20-year ranges. a) Annual QFT conversion rate by age and sex; b) annual TST conversion rate by age and sex; c) annual QFT conversion rate by age and study site; d) annual TST conversion rate by age and study site. 
of IGRA. After 1 year observation, 9084 of them were QFT- and TST-negative, and 90 (0.1\%) persons had conversion by both tests, 1256 (13.8\%) TST conversion alone and 133 (14.6\%) QFT conversion only. Concordance was $86.9 \%$ ( 9174 out of 10563 ) but with a very low $\kappa$ value of 0.08 . With respect to study sites, $\kappa$ values were found to be 0.05 for site $A, 0.08$ for site $B, 0.14$ for site $C$ and 0.10 for site $D$.

\section{Persistence of QFT conversion in the second yearly follow-up}

As shown in figure 1, a total of 390 participants were identified with QFT conversions and 330 (84.6\%) of them completed the second yearly QFT test. Persistent QFT positive results were observed in $49.7 \%$ (166 out of 330 ) on average, with a range of $22.1-74.1 \%$ between the study sites (table 4 and table S3). Increased QFT-positive persistence was observed among conversions with increasing age and family income.

With respect to the status of QFT reversion and persistence, the difference in QFT quantitative values for the definition of conversion at the first year follow-up survey were compared. There was no statistically significant difference observed between the two groups, as shown in figure 3 . However, when the analysis was stratified by age, those with reversions had much lower quantitative values among participants 60 years and older as compared to individuals with persistent positive results $(\mathrm{p}=0.007)$. A similar difference, though not statistically significant, was observed for those participants aged $15-19$ years $(\mathrm{p}=0.121)$.

In the sensitivity analysis, using a more stringent cut-off of $0.70 \mathrm{IU} \cdot \mathrm{mL}^{-1}$ for the conversion definition did not substantially alter the association analysis results for QFT persistence, as shown in table S4.

TABLE 2 Univariate and multivariate analysis of tuberculin skin test (TST) conversion

TST conversion

$741 / 5030(14.73 \%)$

$913 / 6392(14.28 \%)$

Female

\section{Age}

$5-9$ years

$10-19$ years

20-29 years

$30-39$ years

40-49 years

$50-59$ years

60-69 years

$\geqslant 70$ years

Highest education level

Primary school or lower

Middle school

High school

College or higher

Family per capita income

Less than RMB 6000

RMB 6000 or more

Smoking status

Never smoked

Ever smoked

Alcohol drinking

No

Yes

BCG scar

Absent

Present

BMI

$<18.5 \mathrm{~kg} \cdot \mathrm{m}^{-2}$

$\geqslant 18.5-<24 \mathrm{~kg} \cdot \mathrm{m}^{-2}$

$\geqslant 24-<28 \mathrm{~kg} \cdot \mathrm{m}^{-2}$

$\geqslant 28 \mathrm{~kg} \cdot \mathrm{m}^{-2}$

History of close contact with TB patient

No

Yes
$91 / 1112(8.18 \%)$

$106 / 1219(8.70 \%)$

$131 / 819(16.00 \%)$

$137 / 985$ (13.91\%)

$423 / 2475(17.09 \%)$

$315 / 2008(15.69 \%)$

$311 / 1807(17.21 \%)$

140/997 (14.04\%)

$829 / 6697(12.38 \%)$

$619 / 3457$ (17.91\%)

$172 / 1011$ (17.01\%)

$34 / 257$ (13.23\%)

$911 / 8043(13.33 \%)$

$743 / 3379$ (21.99\%)

$1236 / 9004(13.73 \%)$

418/2415 (17.31\%)

$1305 / 9301(14.03 \%)$

$349 / 2120$ (16.46\%)

$852 / 5647$ (15.09\%)

$802 / 5774$ (13.89\%)

$827 / 5157$ (16.04\%)

$192 / 2122$ (9.05\%)

454/2968 (15.30\%)

$181 / 1175$ (15.40\%)

$1557 / 10881$ (14.31\%)

$95 / 521(18.23 \%)$
Crude $0 \mathrm{R}^{\#}(95 \% \mathrm{CI})$

Ref.

0.96 (0.86-1.07)

Ref.

$1.07(0.80-1.43)$

$2.14(1.61-2.84)$

$1.81(1.37-2.40)$

2.31(1.82-2.94)

$2.09(1.63-2.67)$

$2.33(1.82-2.99)$

$1.83(1.39-2.42)$

Ref.

1.54 (1.38-1.73)

$1.45(1.21-1.74)$

$1.08(0.75-1.56)$

Ref.

$2.56(2.32-2.86)$

Ref.

$1.31(1.16-1.49)$

Ref.

$1.21(1.06-1.37)$

Ref.

$0.91(0.81-1.01)$

$0.52(0.44-0.62)$

Ref.

$0.95(0.84-1.07)$

$0.95(0.80-1.14)$

Ref.

$1.34(1.06-1.68)$
Adjusted $\mathrm{OR}^{\#}(95 \% \mathrm{Cl})$

BCG: bacille Calmette-Guérin; BMI: body mass index; TB: tuberculosis. " : controlling for variables with significant association in univariate analysis. 
TABLE 3 Univariate and multivariate analysis of QuantiFERON-TB Gold In-Tube (QFT) conversion

QFT conversion

$218 / 5726(3.81 \%)$

Male

Female

Age

5-9 years

$10-19$ years

20-29 years

$30-39$ years

40-49 years

$50-59$ years

60-69 years

$\geqslant 70$ years

Highest education level

Primary school or lower

Middle school

High school

College or higher

Family per capita income

Less than RMB 6000

RMB 6000 or more

Smoking status

Never smoked

Ever smoked

Alcohol drinking

No

Yes

BCG scar

Absent

Present

BMI

$<18.5 \mathrm{~kg} \cdot \mathrm{m}^{-2}$

$\geqslant 18.5-<24 \mathrm{~kg} \cdot \mathrm{m}^{-2}$

$\geqslant 24-<28 \mathrm{~kg} \cdot \mathrm{m}^{-2}$

$\geqslant 28 \mathrm{~kg} \cdot \mathrm{m}^{-2}$

History of close contact with TB patient

No

Yes
$172 / 6990$ (2.46\%)

$5 / 1181(0.42 \%)$

$21 / 1346(1.56 \%)$

$30 / 1029$ (2.92\%)

$36 / 1250$ (2.88\%)

$105 / 2935$ (3.58\%)

70/2137 (3.28\%)

$78 / 1847$ (4.22\%)

$45 / 991(4.54 \%)$

$204 / 7043$ (2.90\%)

$149 / 4135$ (3.60\%)

$29 / 1213(2.39 \%)$

$8 / 325(2.46 \%)$

$230 / 8503(2.70 \%)$

$160 / 4213$ (3.80\%)

$244 / 9880(2.47 \%)$

$146 / 2833$ (5.15\%)

$294 / 10353(2.84 \%)$

$96 / 2362$ (4.06\%)

$220 / 6749$ (2.96\%)

$186 / 5815$ (3.20\%)

$36 / 2292(1.57 \%)$

120/3341 (3.59\%)

48/1267 (3.79\%)

$360 / 12086(2.98 \%)$

$29 / 609$ (4.76\%)
$190 / 5966$ (3.18\%)
Crude $0 \mathrm{R}^{\#}(95 \% \mathrm{Cl})$

Ref.

$0.63(0.52-0.78)$

Ref.

$3.72(1.40-9.92)$

$7.06(2.73-18.27)$

$6.98(2.73-17.83)$

$8.73(3.55-21.46)$

7.97 (3.21-19.79)

10.37 (4.19-25.69)

$11.19(4.42-28.30)$

Ref.

$1.25(1.01-1.55)$

$0.82(0.55-1.22)$

$0.85(0.41-1.73)$

Ref.

$1.10(0.89-1.36)$

Ref.

$2.15(1.74-2.65)$

Ref.

1.45 (1.15-1.83)

Ref.

$0.91(0.76-1.14)$

$0.48(0.40-0.69)$

Ref.

$1.13(0.89-1.42)$

$1.19(0.86-1.65)$

Ref.

$1.63(1.10-2.40)$
Adjusted OR ${ }^{\#}(95 \% \mathrm{CI})$

Ref.

$0.70(0.53-0.94)$

Ref.

$3.86(1.43-10.44)$

7.22 (2.55-20.47)

$6.74(2.41-18.85)$

8.51 (3.16-22.93)

7.75 (2.86-21.03)

9.89 (3.69-26.51)

$10.87(4.03-29.36)$

Ref.

$1.10(0.86-1.40)$

$0.75(0.49-1.14)$

$0.83(0.39-1.78)$

Ref.

$1.53(1.13-2.05)$

Ref.

$0.80(0.60-1.05)$

$1.13(0.74-1.72)$

Ref.

$1.10(0.87-1.39)$

$1.20(0.87-1.67)$

Ref.

$1.54(1.04-2.27)$

BCG: bacille Calmette-Guérin; BMI: body mass index; TB: tuberculosis. " ${ }^{\text {: }}$ controlling for variables with significant association in univariate analysis.

\section{Discussion}

This is the first ever population-based multicentre prospective study conducted in China to trace the acquisition and persistence of TB infection using serial testing with both IGRA and TST. The important findings of this prospective representative population study include the high self-reported rural rate of TB exposure, as well as the high risk of annual and persistent rates of TB infection, especially among older populations. According to the existing provision, Administrative Measures for Prevention and Control of Tuberculosis (2013), chemoprophylaxis is suggested for HIV-infected and child contacts, 5 years of age on the premise of informed consent [14]. None of the populations identified to be QFT/TST positive in the present study have recommendations for preventive treatment. This study included persons $>5$ years and none had officially registered or self-reported HIV infection. Hence, this study provided a unique opportunity to observe the acquisition and persistence of $\mathrm{TB}$ infection in the study population without influence from LTBI treatment. The variability of rates of acquired infection by geographic location, and parallel rates of LTBI prevalence and conversion to background TB prevalence (based on QFT) have important implications for China and areas with similar profiles [15].

Even if using the minimal estimate of persistent QFT positives after conversion of $1.5 \%$ as the average annual rate of TB infection, $>20$ million people in China would be affected each year. However, application of the $1.5 \%$ conversion rate countrywide has limitations since data from the four study sites may not accurately reflect the general situation of the whole country. As a comparison, the annual risk of TB 
TABLE 4 Univariate and multivariate analysis of QuantiFERON-TB Gold In-Tube (QFT) persistence based on a traditional conversion definition ${ }^{\#}$

\begin{tabular}{|c|c|c|c|}
\hline & QFT persistence & Crude OR $(95 \% \mathrm{CI})$ & Adjusted OR $(95 \% \mathrm{CI})$ \\
\hline Total & $166 / 330(49.70 \%)$ & NA & \\
\hline \multicolumn{4}{|l|}{ Sex } \\
\hline Male & $101 / 183$ (55.19\%) & Ref. & Ref. \\
\hline Female & 63/147 (42.86\%) & $0.61(0.39-0.94)$ & $0.88(0.46-1.67)$ \\
\hline \multicolumn{4}{|l|}{ Age } \\
\hline $5-19$ years & $4 / 24(16.67 \%)$ & Ref. & Ref. \\
\hline $20-29$ years & $7 / 18(38.89 \%)$ & $3.18(0.76-13.32)$ & $2.09(0.44-9.91)$ \\
\hline $30-39$ years & $13 / 28(46.43 \%)$ & 4.33 (1.17-15.98) & $4.00(0.94-16.96)$ \\
\hline 40-49 years & $44 / 88(50.00 \%)$ & $5.00(1.58-15.82)$ & $4.53(1.22-16.86)$ \\
\hline $50-59$ years & $33 / 62$ (53.23\%) & $5.69(1.74-18.58)$ & $5.95(1.55-22.86)$ \\
\hline$\geqslant 60$ years & $63 / 110(57.27 \%)$ & $6.70(2.15-20.91)$ & 7.05 (1.91-26.10) \\
\hline \multicolumn{4}{|l|}{ Highest education level } \\
\hline Primary school or lower & $80 / 178$ (44.94\%) & Ref. & Ref. \\
\hline Middle school & $73 / 127(57.48 \%)$ & $1.66(1.05-2.62)$ & $2.11(1.21-3.68)$ \\
\hline High school & $10 / 21(47.62 \%)$ & $1.11(0.45-2.76)$ & $0.97(0.35-2.66)$ \\
\hline College or higher & $1 / 4(25.00 \%)$ & $0.41(0.04-4.00)$ & $0.57(0.05-6.83)$ \\
\hline \multicolumn{4}{|l|}{ Family per capita income } \\
\hline Less than RMB 6000 & $83 / 205(40.49 \%)$ & Ref. & Ref. \\
\hline RMB 6000 or more & $81 / 125$ (64.80\%) & $2.35(1.48-3.74)$ & $2.15(1.30-3.54)$ \\
\hline \multicolumn{4}{|l|}{ Smoking status } \\
\hline Never smoked & $89 / 204$ (43.63\%) & Ref. & Ref. \\
\hline Ever smoked & $75 / 126(59.52 \%)$ & $1.90(1.21-2.98)$ & $1.39(0.73-2.63)$ \\
\hline \multicolumn{4}{|l|}{ Alcohol drinking } \\
\hline No & $120 / 243(49.38 \%)$ & Ref. & \\
\hline Yes & 44/87 (50.57\%) & $1.05(0.64-1.71)$ & \\
\hline \multicolumn{4}{|l|}{ BCG scar } \\
\hline Absent & $76 / 165$ (46.06\%) & Ref. & \\
\hline Present & $88 / 165$ (5.33\%) & $1.34(0.87-2.06)$ & \\
\hline \multicolumn{4}{|l|}{ BMI } \\
\hline$<18.5 \mathrm{~kg} \cdot \mathrm{m}^{-2}$ & $10 / 31$ (32.26\%) & $0.37(0.16-0.83)$ & $1.14(0.42-3.06)$ \\
\hline$\geqslant 18.5-<24 \mathrm{~kg} \cdot \mathrm{m}^{-2}$ & $87 / 154(56.49 \%)$ & Ref. & Ref. \\
\hline$\geqslant 24-<28 \mathrm{~kg} \cdot \mathrm{m}^{-2}$ & $50 / 108$ (46.30\%) & $0.66(0.41-1.09)$ & $1.63(0.96-2.77)$ \\
\hline$\geqslant 28 \mathrm{~kg} \cdot \mathrm{m}^{-2}$ & $17 / 37$ (45.95\%) & $0.66(0.32-1.35)$ & $1.53(0.70-3.33)$ \\
\hline
\end{tabular}

BCG: bacille Calmette-Guérin; BMI: body mass index. ${ }^{\#}$ : using a cut-off of $\geqslant 0.35 \mathrm{IU} \cdot \mathrm{mL}^{-1}$; ${ }^{\text {9 }}$ : controlling for variables with significant association in univariate analysis.

infection had been estimated at $1-2 \%$ for most of the tuberculin surveys carried out in different areas over different time periods in India, a country with the highest TB burden in the world [16]. In Africa, a number of tuberculin surveys have been published, and annual risks of TB infection between $0.3 \%$ and $3.1 \%$ have been reported [17-19]. In China, a country with much lower TB incidence than India and most African countries, the incidence of annual TB infection was previously estimated at $1.3 \%$ for children between age 0-14 years based on the nationwide TB epidemiology survey conducted in 2000 [20]. As compared to this paediatric survey estimate, the annual risk of infection (ARI) of our present study is much higher if based on QFT conversion (average 3.1\%) but it is similar if based on QFT conversion persistence (average 1.5\%). In contrast to QFT, the $14.5 \%$ ARI based on TST conversion far exceeded the estimated ARIs of the highest burden countries of the world and could not be explained by background TB rates of the rural sites. The booster phenomenon as a likely cause, has been well described in serial testing [21,22]. Considering such a limitation of TST, persistent infection was only addressed for QFT in the present study.

Surprisingly, being elderly ( $\geqslant 60$ years) was associated with recent TB infection as defined by both QFT and TST conversion. Elderly subjects ( $\geqslant 60$ years old) showed higher QFT conversion as compared to the younger cohorts (4.3\% versus $2.7 \%$ ) and higher QFT positivity persistence rates (57.3\% versus $45.9 \%)$, respectively. These findings, together with our published LTBI prevalence data [10], provide stronger population-based evidence, for the first time, that the rural elderly of China carry a double burden of both a high prevalence of LTBI and higher risk of new infection. Ageing and chronic diseases related to attenuation of immunity might explain the higher susceptibility of the elderly population to infection and reinfection. Modelling analysis suggested preventative therapy for elderly populations would enable China 


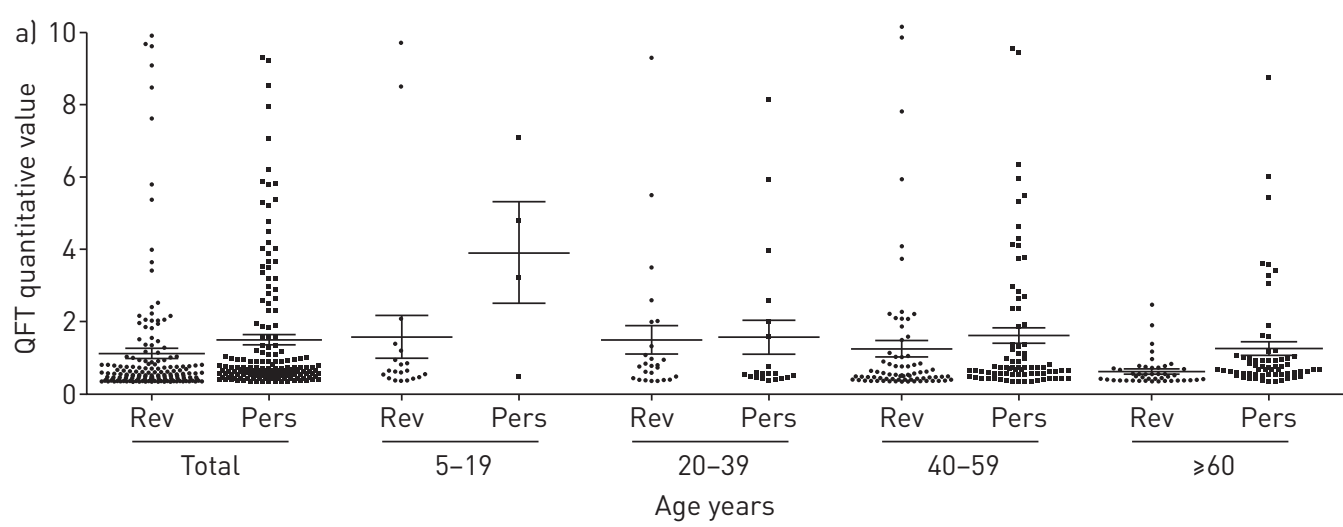

\begin{tabular}{rccccc} 
b) & Total & \multicolumn{4}{c}{ Age years } \\
\cline { 3 - 6 } & & $5-19$ & $20-39$ & $40-59$ & $\geqslant 60$ \\
\hline QFT quantitative value & & & & & \\
Persistent group & $1.498 \pm 0.144$ & $3.880 \pm 1.384$ & $1.533 \pm 0.462$ & $1.587 \pm 0.219$ & $1.222 \pm 0.187$ \\
& $(\mathrm{n}=163)$ & $(\mathrm{n}=4)$ & $(\mathrm{n}=20)$ & $(\mathrm{n}=77)$ & $(\mathrm{n}=62)$ \\
Reversion group & $1.131 \pm 0.136$ & $1.557 \pm 0.584$ & $1.467 \pm 0.378$ & $1.224 \pm 0.221$ & $0.618 \pm 0.057$ \\
& $(\mathrm{n}=166)$ & $(\mathrm{n}=20)$ & $(\mathrm{n}=26)$ & $(\mathrm{n}=73)$ & $(\mathrm{n}=47)$ \\
Mean difference & 0.367 & 2.324 & 0.065 & 0.362 & 0.605 \\
p-value for t-test & 0.064 & 0.121 & 0.913 & 0.245 & 0.007 \\
\hline
\end{tabular}

FIGURE 3 The distribution of QuantiFERON-TB Gold In-Tube (QFT) quantitative values among conversions by later persistence status. With respect to the status of conversion persistence, the distribution of QFT quantitative values among the conversions was not statistically different. a) Individual data. Rev: reversed; Pers: persistent. b) Summarised data. Data are presented as meanıSEM unless otherwise stated.

to reduce TB incidence by $84 \%$ (78-93\%) [23]. Furthermore, our results support contact investigation and treatment of infected close contacts of any age, including the elderly [24, 25]. The persistence of QFT positivity was $49.7 \%$, and positively associated with old age and family income. Clearance of infection by host immunity is believed to be a reason for reversion. However, previous studies performed in the healthcare workers from the USA, a country with very low TB incidence, persistence of QFT positivity has been reported to be as low as $10-35 \%$, implying the majority being due to false conversions [26, 27]. In contrast, the significantly higher rate of QFT-positive persistence found in this study would be expected in a high-burden country [27]. The difference of persistent positivity is likely to be attributed to the higher positive predictive value in populations with true higher exposure and infection prevalence. In addition, QFT conversion using a nonstringent dichotomous cut-point should be interpreted carefully. Quantitative values of older participants ( $>60$ years older) with persistent positive results versus reversions on the third QFT test, significantly differed and were lower among those with test reversion. The mean quantitative values of the second QFT test among the elderly were also lower than younger cohorts with a distinct correlation with age. It suggests that older individuals may have quantitative values that may vary around the cut-point and a higher cut-off value for conversion definition might be appropriate. However, in our sensitivity analysis using a hypothetical higher cut-off value $\left(0.70 \mathrm{IU} \cdot \mathrm{mL}^{-1}\right)$ to define conversion $[13,28]$, older age consistently correlated to higher rates of persistent positive QFT results. Without a gold standard for LTBI, cut-off values for QFT conversion cannot be ascertained as the only contributor to the high reversion. The attenuation of immunity among the older population might predict a low infection clearance capability compared to younger cohorts and higher risk of persistent infection.

Another important finding of this series of studies in China is the examination of the TST and QFT as surveillance tools. In addition to the implausibly high TST-based ARI, we urge caution in the interpretation of TST conversion results in individuals who are BCG vaccinated, reside in nontuberculous Mycobacterium (NTM)-prevalent areas and are elderly. Previous estimates of ARI were historically based on TST and might indicate overestimation [29]. Of note, the TST conversion rate was highest at site A where the incidence of TB was the lowest among all of the sites. This finding was consistent with the prior prevalence data and supports our hypotheses that TST results at site A might be influenced by its location in the coastal region in east China, an area which may have higher NTM exposure [10]. In addition, as shown in figure 1, the annual TST reversion rate was as high as $77.5 \%$ among those QFT-negative, TST-positive subjects at baseline, again, suggesting poor specificity. Therefore, although there is a lack of 
gold-standard method to detect TB infection, the longitudinal data provide compelling evidence that the TST is a poor surveillance tool in China where BCG practices have changed over time and when the background of common NTM infection rates has not been established.

When interpreting the results of the present study, there are limitations. First, as our previous study suggested, age and BCG vaccination status should be taken into consideration in deciding an appropriate TST cut-off. The current cut-offs used for TST positivity at baseline and of TST conversion at follow-up do not appear to minimise false-positive or boosted responses from BCG or environmental mycobacteria. The high TST reversion rate of $77.53 \%$ among those with discordant QFT-negative, TST-positive baseline results are highly suggestive of a large number of false-positive results. In regards to QFT conversion, the dichotomous cut-point of $0.35 \mathrm{IU} \cdot \mathrm{mL}^{-1}$ may also result in an overestimation of converters for individuals with baseline negative results that are close to the cut-point [11]. Although less reversion compared to the TST was observed (41\%) among those with discordant QFT-negative, TST-positive baseline results, further analysis of quantitative results are needed. Second, differences in demographics across the study sites make the comparison between study sites difficult. However, when active TB and LTBI epidemiology are combined with the population characteristics and health infrastructure of each site, the interpretation of the new infection rate appears consistent [10]. See tables S5-7 for the results where study site was included in the association analyses as a variable. Third, to avoid a potential boosting effect of TST on the QFT result, QFT was performed prior to TST. However, we could not exclude the possibility that serial TST might have boosted QFT results even of the interval between the serial tests was 1 year. Facing the fact that there is no gold standard for LTBI testing, except for developing more advanced tools, further studies are needed to evaluate and to improve the existing methods [30].

In summary, this population-based multicentre cohort study tracked TB infection (using both IGRA and TST) and strongly suggests that more attention should be drawn to the higher than expected TB transmission in rural areas of China. The elderly were found to be a promising target population for TB infection control and LTBI management in rural China due to the higher acquisition and persistence of infection found in this study.

\section{Acknowledgements}

We thank Shuigao Jin and Lixia Wang from the Chinese Center for Disease Prevention and Control (Beijing, China), and Yude Chen from Peking University (Beijing, China) for their help with study implementation. We thank all the investigators from the study sites for their contribution to the site work at the baseline survey and follow-up examinations.

Q. Jin designed the study. L. Gao, W. Xu and X. Chen organized the implementation of the study. L. Bai, J. He, W. Lu and J. Liu organised survey in the study sites. X. Lu, J. Du, H. Zhang, H. Su, M. Li, B. Feng, Z. Li, H. Xin, H. Sui and H. Li performed study site coordination and field work quality control. X. Lu, J. Du and Y. Xia were in charge of data management. X. Wang, F. Shen, J. He, S. Yang, H. Su, Y. Wang, Z. Xu, Y. Tan, T. Chen, H. Peng, Z. Wang, T. Zhu and W. Xu studied participants' follow-up investigations and tests. Y. Ma and Z. Zhang organised the reading of chest radiographs. L. Gao, Q. Jin and W. Xu performed data analysis and wrote the manuscript. S. Chen, X. Chen, A. Catanzaro, C. Chen and T.G. Evans commented on the report and improved the English writing. All authors contributed to revision, and saw and approved the final version of the manuscript.

\section{References}

1 Andrews JR, Noubary F, Walensky RP, et al. Risk of progression to active tuberculosis following reinfection with Mycobacterium tuberculosis. Clin Infect Dis 2012; 54: 784-791.

Zumla A, Raviglione M, Hafner R, et al. Tuberculosis. N Engl J Med 2013; 368: 745-755.

Lobue P, Menzies D. Treatment of latent tuberculosis infection: an update. Respirology 2010; 15: 603-622.

World Health Organization. Guidelines on the management of latent tuberculosis infection. Geneva, WHO, 2014.

National Tuberculosis Controllers Association, Centers for Disease Control and Prevention. Targeted tuberculin testing and treatment of latent tuberculosis infection. MMWR Recomm Rep 2000; 49: 1-51.

6 National Collaborating Centre for Chronic Conditions (UK), Centre for Clinical Practice at NICE (UK). Tuberculosis: Clinical Diagnosis and Management of Tuberculosis, and Measures for Its Prevention and Control. London, NICE, 2011.

7 World Health Organization. Global Tuberculosis Report 2014. Geneva, WHO, 2014

8 Lönnroth K, Migliori GB, Abubakar I, et al. Towards tuberculosis elimination: an action framework for low-incidence countries. Eur Respir J 2015; 45: 928-952.

9 D'Ambrosio L, Dara M, Tadolini M, et al. Tuberculosis elimination: theory and practice in Europe. Eur Respir J 2014; 43: 1410-1420.

10 Gao L, Lu W, Bai LQ, et al. Latent tuberculosis infection in rural China: baseline results of a population-based, multicentre, prospective cohort study. Lancet Infect Dis 2015; 15: 310-319.

11 Mazurek GH, Jereb J, Vernon A, et al. Updated guidelines for using interferon gamma release assays to detect Mycobacterium tuberculosis infection - United States, 2010. MMWR Recomm Rep 2010; 59: 1-25.

12 Cheng SM, Wang G, Wang LX. Guidance of Tuberculin Skin Test. Beijing, People's Medical Publishing House, 2014.

13 Pai M, Joshi R, Dogra S, et al. Serial testing of health care workers for tuberculosis using interferon- $\gamma$ assay. Am J Respir Crit Care Med 2006; 174: 349-355.

14 National Health and Family Planning Commission of China. Administrative Measures for Prevention and Control of Tuberculosis. www.moh.gov.cn/mohzcfgs/s3576/201303/727b973d5ee4403ead07e1a2a22928f6.shtml Date last updated: 2013.

15 Rieder H. Annual risk of infection with Mycobacterium tuberculosis. Eur Respir J 2005; 25: 181-185. 


\section{Ibiary S, Coster EJ, Tolba FM, et
Tuberc Lung Dis 1999; 3: 294-299.}

19 Kritzinger FE, Boon S, Verver S, et a Cape Town, South Africa. Trop Med Int Health 2009; 14: 136-142.

20 He GX, Wang L, Du X, et al. Evaluation of the risks for tuberculosis infection and transmission. Chin J Public Health 2003; 7: 867-868.

21 Sepulveda RL, Ferrer X, Latrach C, et al. The influence of Calmette-Guérin bacillus immunization on the booster effect of tuberculin testing in healthy young adults. Am Rev Respir Dis 1990; 142: 24-28.

22 Menzies D. Interpretation of repeated tuberculin tests. Boosting, conversion, and reversion Am J Respir Crit Care Med 1999; 159: 15-21.

23 Huynh GH, Klein DJ, Chin DP, et al. Tuberculosis control strategies to reach the 2035 global targets in China: the role of changing demographics and reactivation disease. BMC Med 2015; 13: 88.

24 Wang L, Zhang H, Ruan Y, et al. Tuberculosis prevalence in China, 1990-2010; a longitudinal analysis of national survey data. Lancet 2014; 383: 2057-2064.

25 Raviglione M, Marais B, Floyd K, et al. Scaling up interventions to achieve global tuberculosis control: progress and new developments. Lancet 2012; 379: 1902-1993.

26 Joshi M, Monson TP, Joshi A, et al. IFN- $\gamma$ release assay conversions and reversions. Challenges with serial testing in U.S. health care workers. Ann Am Thorac Soc 2014; 11: 296-302.

27 Slater ML, Welland G, Pai M, et al. Challenges with QuantiFERON-TB Gold assay for large-scale, routine screening of U.S. healthcare workers. Am J Respir Crit Care Med 2013; 188: 1005-1010.

28 Ringshausen FC, Nienhaus A, Schablon A, et al. Predictors of persistently positive Mycobacterium-tuberculosisspecific interferon $\gamma$ responses in the serial testing of health care workers. BMC Infect Dis 2010; 10: 220.

29 Getahun H, Matteelli A, Abubakar I, et al. Management of latent Mycobacterium tuberculosis infection: WHO guidelines for low tuberculosis burden countries. Eur Respir J 2015; 46: 1563-1576.

30 Barcellini L, Borroni E, Brown J, et al. First independent evaluation of QuantiFERON-TB Plus performance. Eur Respir J 2016; 47: 1587-1590. 